\title{
On perfect packings in dense graphs
}

\author{
József Balogh, ${ }^{*}$ Alexandr V. Kostochka ${ }^{\dagger}$ and Andrew Treglown ${ }^{\ddagger}$
}

Submitted: Jun 30, 2011; Accepted: Mar 2, 2013; Published: Mar 8, 2013

Mathematics Subject Classifications: 05C15, 05C35, 05C70

\begin{abstract}
We say that a graph $G$ has a perfect $H$-packing if there exists a set of vertexdisjoint copies of $H$ which cover all the vertices in $G$. We consider various problems concerning perfect $H$-packings: Given $n, r, D \in \mathbb{N}$, we characterise the edge density threshold that ensures a perfect $K_{r}$-packing in any graph $G$ on $n$ vertices and with minimum degree $\delta(G) \geqslant D$. We also give two conjectures concerning degree sequence conditions which force a graph to contain a perfect $H$-packing. Other related embedding problems are also considered. Indeed, we give a structural result concerning $K_{r}$-free graphs that satisfy a certain degree sequence condition.
\end{abstract}

\section{Introduction}

Given two graphs $H$ and $G$, a perfect $H$-packing in $G$ is a collection of vertex-disjoint copies of $H$ which cover all the vertices in $G$. Perfect $H$-packings are also referred to as $H$-factors or perfect $H$-tilings. Hell and Kirkpatrick [8] showed that the decision problem whether a graph $G$ has a perfect $H$-packing is NP-complete precisely when $H$ has a component consisting of at least 3 vertices. So for such graphs $H$, it is unlikely that there is a complete characterisation of those graphs containing a perfect $H$-packing. Thus, there has been significant attention on obtaining sufficient conditions that ensure a graph $G$ contains a perfect $H$-packing.

A seminal result in the area is the Hajnal-Szemerédi theorem [7] which states that a graph $G$ whose order $n$ is divisible by $r$ has a perfect $K_{r}$-packing provided that $\delta(G) \geqslant$ $(r-1) n / r$. Kühn and Osthus $[12,13]$ characterised, up to an additive constant, the

${ }^{*}$ University of Illinois, Urbana-Champaign, USA and University of California, San Diego, USA, jobal@math.uiuc.edu. This author is supported by NSF CAREER Grant DMS-0745185, UIUC Campus Research Board Grant 11067, and OTKA Grant K76099.

${ }^{\dagger}$ University of Illinois, Urbana-Champaign, USA and Institute of Mathematics, Novosibirsk, Russia, kostochk@math.uiuc.edu. This author is supported in part by NSF grant DMS-0965587 and by grant 12-01-00631 of the Russian Foundation for Basic Research.

${ }^{\ddagger}$ Queen Mary, University of London, United Kingdom, treglown@maths.qmul.ac.uk 
minimum degree which ensures a graph $G$ contains a perfect $H$-packing for an arbitrary graph $H$.

It is easy to see that the minimum degree condition in the Hajnal-Szemerédi theorem cannot be lowered. Of course, this does not mean that one cannot strengthen this result. Ore-type degree conditions consider the sum of the degrees of non-adjacent vertices in a graph. The following Ore-type result of Kierstead and Kostochka [10] implies the HajnalSzemerédi theorem.

Theorem 1 (Kierstead and Kostochka [10]). Let $n, r \in \mathbb{N}$ such that $r$ divides $n$. Suppose that $G$ is a graph on $n$ vertices such that for all non-adjacent $x \neq y \in V(G)$,

$$
d(x)+d(y) \geqslant 2(1-1 / r) n-1 .
$$

Then $G$ contains a perfect $K_{r}$-packing.

Kühn, Osthus and Treglown [14] characterised, asymptotically, the Ore-type degree condition which ensures a graph $G$ contains a perfect $H$-packing for an arbitrary graph $H$.

\subsection{Perfect packings in dense graphs of low minimum degree}

It is easy to characterise the edge density that forces a graph $G$ to contain a perfect $K_{r}$-packing when there are no other restrictions. Indeed, given $n, r \in \mathbb{N}$ such that $r \geqslant 2$ divides $n$, if $G$ is a graph on $n$ vertices and $e(G) \geqslant\left(\begin{array}{l}n \\ 2\end{array}\right)-n+r$ then $G$ contains a perfect $K_{r}$-packing. Moreover, if $G$ is a copy $K$ of $K_{n-1}$ together with a vertex which sends precisely $r-2$ edges to $K$, then $e(G)=\left(\begin{array}{l}n \\ 2\end{array}\right)-n+r-1$ and $G$ does not contain a perfect $K_{r}$-packing. The following result of Akiyama and Frankl [1] refines this observation.

Theorem 2 (Akiyama and Frankl [1]). Let $n, r \in \mathbb{N}$ such that $r$ divides $n$. Suppose $G$ is a graph on $n$ vertices and $e(\bar{G}) \leqslant \min \left\{\left(\begin{array}{c}n / r+1 \\ 2\end{array}\right), n-r+1\right\}$. Then $G$ has a perfect $K_{r}$-packing unless $\bar{G}$ is isomorphic to one of the following graphs:

(i) A copy of $K_{n / r+1}$ together with $(1-1 / r) n-1$ isolated vertices;

(ii) The disjoint union of $K_{1, n-r-j+1}, j$ edges and $r-j-2$ isolated vertices, for some $1 \leqslant j \leqslant r-2$.

When (for example) $n \geqslant r^{3},\left(\begin{array}{c}n / r+1 \\ 2\end{array}\right)>n-r+1$. Hence, in this case Theorem 2 is equivalent to the following: If $G$ is a graph on $n$ vertices and $e(G) \geqslant\left(\begin{array}{l}n \\ 2\end{array}\right)-n+r-1$ then either $G$ contains a perfect $K_{r}$-packing or $\bar{G}$ is isomorphic to a graph as in (ii).

In Sections 2 and 3 we consider the following natural problem: Let $n, r \in \mathbb{N}$ such that $r$ divides $n$. Given some $D \in \mathbb{N}$, what edge density condition ensures that any graph $G$ on $n$ vertices and of minimum degree $\delta(G) \geqslant D$ contains a perfect $K_{r}$-packing?

We fully resolve the problem, and our answers for $r=2$ and $r \geqslant 3$ differ. 
Theorem 3. For an even positive $n$ and integer $1 \leqslant d<n / 2$, let $h(n, d):=\left(\begin{array}{c}n-d-1 \\ 2\end{array}\right)+$ $d(d+1)$ and let $f(2, n, d)$ denote the maximum integer $c$ such that some $n$-vertex graph with minimum degree at least $d$ and at least $c$ edges has no perfect matching. Then

$$
f(2, n, d)=\max \{h(n, d), h(n, 0.5 n-1)\} .
$$

Theorem 4. Let $n, r \in \mathbb{N}$ such that $r \geqslant 3$ and $r$ divides $n$. Given any $D \in \mathbb{N}$ such that $r-1 \leqslant D \leqslant(r-1) n / r-1$ define

$$
g(n, r, D):=\max \left\{\left(\begin{array}{l}
n \\
2
\end{array}\right)-\left(\begin{array}{c}
n / r+1 \\
2
\end{array}\right), D(n-D)+\left(\begin{array}{c}
n-1-D \\
2
\end{array}\right)+e(T(D, r-2))\right\} .
$$

Suppose that $G$ is a graph on $n$ vertices with $\delta(G) \geqslant D$ and $e(G)>g(n, r, D)$. Then $G$ contains a perfect $K_{r}$-packing. Moreover, there exists a graph $G^{\prime}$ on $n$ vertices with $\delta\left(G^{\prime}\right) \geqslant D$ and $e\left(G^{\prime}\right)=g(n, r, D)$ but such that $G^{\prime}$ does not contain a perfect $K_{r}$-packing.

Clearly a graph $G$ of minimum degree $\delta(G)<r-1$ cannot contain a perfect $K_{r^{-}}$ packing. Further, regardless of edge density, every graph $G$ whose order $n$ is divisible by $r$ and with $\delta(G) \geqslant(r-1) n / r$ contains a perfect $K_{r}$-packing. Thus, Theorem 4 covers all values of $D$ where our problem was not solved previously.

An equitable $k$-colouring of a graph $G$ is a proper $k$-colouring of $G$ such that any two colour classes differ in size by at most one. Let $n, r \in \mathbb{N}$ such that $r$ divides $n$. Notice that a graph $G$ on $n$ vertices has a perfect $K_{r}$-packing if and only if the complement $\bar{G}$ of $G$ has an equitable $n / r$-colouring. So, for example, the Hajnal-Szemerédi theorem can be stated in terms of equitable colourings: Let $G$ be a graph on $n$ vertices such that $r$ divides $n$. If $\Delta(G) \leqslant n / r-1$ then $G$ has an equitable $n / r$-colouring.

It is often easier to work in the language of equitable colourings compared to perfect packings. Indeed, rather than prove Theorem 1 directly, Kierstead and Kostochka proved the equivalent statement for equitable colourings. Here we also find it more convenient to work with equitable colourings. Thus, instead of proving Theorem 4 directly we prove the following equivalent result.

Theorem 5. Let $n, r \in \mathbb{N}$ such that $r \geqslant 3$ and $r$ divides $n$. Recall that $T(n, r)$ denotes the Turán graph. Given any $D \in \mathbb{N}$ such that $n / r \leqslant D \leqslant n-r$ define

$$
f(n, r, D):=\min \left\{\left(\begin{array}{c}
n / r+1 \\
2
\end{array}\right), D+e(\bar{T}(n-D-1, r-2))\right\} .
$$

Suppose that $G$ is a graph on $n$ vertices with $\Delta(G) \leqslant D$ and $e(G)<f(n, r, D)$. Then $G$ has an equitable $n / r$-colouring. Moreover, there exists a graph $G^{\prime}$ on $n$ vertices with $\Delta\left(G^{\prime}\right) \leqslant D$ and $e\left(G^{\prime}\right)=f(n, r, D)$ but such that $G^{\prime}$ does not have an equitable $n / r$ colouring.

We prove Theorem 3 and describe extremal constructions for Theorems 4 and 5 in Section 2. That is, we show that the edge density condition in Theorem 4 is best possible for all values of $D$ such that $r-1 \leqslant D \leqslant(r-1) n / r-1$. Section 3 contains a proof of Theorem 5. 


\subsection{Degree sequence conditions forcing a perfect packing}

Chvátal [3] gave a condition on the degree sequence of a graph which ensures Hamiltonicity: Suppose that $G$ is a graph on $n$ vertices and that the degrees of the graph are $d_{1} \leqslant \ldots \leqslant d_{n}$. If $n \geqslant 3$ and $d_{i} \geqslant i+1$ or $d_{n-i} \geqslant n-i$ for all $i<n / 2$ then $\mathrm{G}$ is Hamiltonian. The following is a simple consequence of Chvátal's theorem.

Theorem 6 (Chvátal [3]). Suppose that $G$ is a graph on $n \geqslant 2$ vertices and the degrees of the graph are $d_{1} \leqslant \ldots \leqslant d_{n}$. If

$$
d_{i} \geqslant i \text { or } d_{n-i+1} \geqslant n-i \text { for all } 1 \leqslant i \leqslant n / 2
$$

then $G$ contains a Hamilton path.

We propose the following conjecture on the degree sequence of a graph which forces a perfect $K_{r}$-packing.

Conjecture 7. Let $n, r \in \mathbb{N}$ such that $r$ divides $n$. Suppose that $G$ is a graph on $n$ vertices with degree sequence $d_{1} \leqslant \ldots \leqslant d_{n}$ such that:

( $\alpha) d_{i} \geqslant(r-2) n / r+i$ for all $i<n / r$;

(ß) $d_{n / r+1} \geqslant(r-1) n / r$.

Then $G$ contains a perfect $K_{r}$-packing.

Note that Conjecture 7, if true, is much stronger than the Hajnal-Szemerédi theorem since the degree condition allows for $n / r$ vertices to have degree less than $(r-1) n / r$. Further, Proposition 17 in Section 4 shows that the condition on the degree sequence in Conjecture 7 is essentially "best possible". It is easy to see that Theorem 6 implies Conjecture 7 in the case when $r=2$. We prove the conjecture in the case when $G$ is additionally $K_{r+1}$-free (see Section 5 ).

If one can prove Conjecture 7 , it seems likely it can be used to prove the next conjecture.

Conjecture 8. Suppose $\gamma>0$ and $H$ is a graph with $\chi(H)=r$. Then there exists an integer $n_{0}=n_{0}(\gamma, H)$ such that the following holds. If $G$ is a graph whose order $n \geqslant n_{0}$ is divisible by $|H|$, and whose degree sequence $d_{1} \leqslant \ldots \leqslant d_{n}$ satisfies

- $d_{i} \geqslant(r-2) n / r+i+\gamma n$ for all $i<n / r$,

then $G$ contains a perfect $H$-packing.

Since first submitting this paper, the third author and Knox [11] have proven Conjecture 8 in the case when $r=2$. (In fact, they have proven a much more general result concerning embedding spanning bipartite graphs of small bandwidth.)

The following result of Erdős [6] characterises those degree sequences which force a copy of $K_{r}$ in a graph $G$. 
Theorem 9 (Erdős $[6]$ ). Let $G$ be a graph on $n$ vertices with degree sequence $d_{1} \leqslant \ldots \leqslant d_{n}$. If $G$ is $K_{r+1}$-free then there is an r-partite graph $G^{\prime}$ on $n$ vertices whose degree sequence $d_{1}^{\prime} \leqslant \ldots \leqslant d_{n}^{\prime}$ satisfies

$$
d_{i} \leqslant d_{i}^{\prime} \text { for all } i \leqslant n .
$$

In Section 6 we prove the following related structural theorem.

Theorem 10. Suppose that $n, r \in \mathbb{N}$ such that $n \geqslant r$ and so that $r$ divides $n$. Let $G$ be a $K_{r+1}$-free graph on $n$ vertices whose degree sequence $d_{1} \leqslant \ldots \leqslant d_{n}$ is such that $d_{n / r} \geqslant(r-1) n / r$. Then $G \subseteq T(n, r)$, where $T(n, r)$ is the complete r-partite Turán graph on $n$ vertices; so each vertex class has size $\lceil n / r\rceil$ or $\lfloor n / r\rfloor$.

\section{The case $r=2$ and extremal examples for $r \geqslant 3$}

\subsection{Perfect matchings in dense graphs}

In this section we establish the density threshold that ensures every graph $G$ on an even number $n$ of vertices and of minimum degree $\delta(G) \geqslant d$ contains a perfect matching. Note that we only consider values of $d$ such that $1 \leqslant d<n / 2$, since if $\delta(G) \geqslant n / 2$ then $G$ has a perfect matching, regardless of the edge density.

Recall that $h(n, d):=\left(\begin{array}{c}n-d-1 \\ 2\end{array}\right)+d(d+1)$. Note that for a fixed even $n, h(n, d)$ decreases with $d$ in the interval $[0, n / 3-5 / 6]$ and increases with $d$ in $[n / 3-5 / 6,0.5 n-1]$.

For a positive even $n$ and an integer $0 \leqslant d<n / 2$, let $A, B$ and $C$ be disjoint sets with $|A|=d+1,|B|=d,|C|=n-2 d-1$. Let $H=H(n, d)$ be the graph with the vertex set $A \cup B \cup C$ such that $H[B \cup C]=K_{n-d-1}$, and each vertex in $A$ is adjacent to each vertex in $B$ and to no vertex in $C$. So $H$ does not contain a perfect matching and has exactly $h(n, d)$ edges.

The examples of $H(n, d)$ show that $f(2, n, d) \geqslant \max \{h(n, d), h(n, 0.5 n-1)\}$. Thus to derive Theorem 3, it suffices to prove that an $n$-vertex graph $G$ with $\delta(G) \geqslant d$ and $e(G)>\max \{h(n, d), h(n, 0.5 n-1)\}$ has a perfect matching.

Consider such a graph $G$. Let $d_{1} \leqslant \ldots \leqslant d_{n}$ denote the degree sequence of $G$. If $d_{i} \geqslant i$ for all $1 \leqslant i \leqslant n / 2$ then Theorem 6 implies that $G$ contains a perfect matching. Suppose for a contradiction that $d_{i^{\prime}} \leqslant i^{\prime}-1$ for some $1 \leqslant i^{\prime} \leqslant n / 2$. Note that $i^{\prime}>d$ as $\delta(G) \geqslant d$.

Let $A$ denote the set of $i^{\prime}$ vertices in $G$ that correspond to the first $i^{\prime}$ terms $d_{1}, \ldots, d_{i^{\prime}}$ of the degree sequence. Set $B:=V(G) \backslash A$. Then

$$
e(G[B]) \geqslant e(G)-i^{\prime}\left(i^{\prime}-1\right)>\max \{h(n, d), h(n, 0.5 n-1)\}-i^{\prime}\left(i^{\prime}-1\right)
$$

since $d(x) \leqslant i^{\prime}-1$ for all $x \in A$. Note that $\max \{h(n, d), h(n, 0.5 n-1)\} \geqslant h\left(n, i^{\prime}-1\right)$ since $d<i^{\prime} \leqslant n / 2$. Therefore,

$$
e(G[B])>\max \{h(n, d), h(n, 0.5 n-1)\}-i^{\prime}\left(i^{\prime}-1\right) \geqslant h\left(n, i^{\prime}-1\right)-i^{\prime}\left(i^{\prime}-1\right)=\left(\begin{array}{c}
n-i^{\prime} \\
2
\end{array}\right)
$$

a contradiction as $|B|=n-i^{\prime}$. Thus, $d_{i} \geqslant i$ for all $1 \leqslant i \leqslant n / 2$, as desired. 


\subsection{Examples for $r \geqslant 3$}

We will give the extremal examples for Theorem 5. Since Theorems 4 and 5 are equivalent, the complements of the extremal graphs for Theorem 5 are the extremal graphs for Theorem 4 .

Proposition 11. Suppose that $n, r \in \mathbb{N}$ such that $r \geqslant 3$ and $r$ divides $n$. Then there exists a graph $G_{1}$ on $n$ vertices such that $\Delta\left(G_{1}\right)=n / r$,

$$
e\left(G_{1}\right)=\left(\begin{array}{c}
n / r+1 \\
2
\end{array}\right)
$$

but such that $G_{1}$ does not have an equitable $n / r$-colouring.

Proof. Let $G_{1}$ denote the disjoint union of a clique $V$ on $n / r+1$ vertices and an independent set $W$ of $(1-1 / r) n-1$ vertices. So every independent set in $G_{1}$ contains at most one vertex from $V$. But since $|V|=n / r+1, G_{1}$ does not have an equitable $n / r$-colouring. Further, $\Delta\left(G_{1}\right)=n / r$ and $e\left(G_{1}\right)=\left(\begin{array}{c}n / r+1 \\ 2\end{array}\right)$.

Proposition 12. Suppose that $n, r \in \mathbb{N}$ such that $r \geqslant 3$ and $n=k r$ for some $k \geqslant 2$. Further, let $D \in \mathbb{N}$ such that $n /(r-1) \leqslant D \leqslant n-r$. Then there exists a graph $G_{2}$ on $n$ vertices such that $\Delta\left(G_{2}\right)=D$,

$$
e\left(G_{2}\right)=D+e(\bar{T}(n-D-1, r-2)),
$$

but such that $G_{2}$ does not have an equitable $n / r$-colouring.

Proof. Let $G_{2}$ denote the disjoint union of a copy $K$ of $K_{1, D}$ and a copy of $\bar{T}(n-D-1, r-$ $2)$. So $|G|=n$. Let $v$ denote the vertex of degree $D$ in $K$. The largest independent set in $G_{2}$ that contains $v$ is of size $r-1$. Thus, $G_{2}$ does not have an equitable $n / r$-colouring. Further, $e\left(G_{2}\right)=D+e(\bar{T}(n-D-1, r-2))$.

Since $n /(r-1) \leqslant D$ we have that $n-1 \leqslant(r-1) D$. Thus, every vertex in the copy of $\bar{T}(n-D-1, r-2)$ has degree at most

$$
\left\lceil\frac{n-D-1}{r-2}\right\rceil-1 \leqslant \frac{n-D-1}{r-2} \leqslant D .
$$

This implies that $\Delta\left(G_{2}\right)=D$.

Clearly Propositions 11 and 12 show that one cannot lower the edge density condition in Theorem 5 in the case when $n /(r-1) \leqslant D \leqslant n-r$. The following result, together with Proposition 11, shows that Theorem 5 is best possible in the case when $n / r \leqslant D \leqslant$ $n /(r-1)$.

Proposition 13. Let $n, r \in \mathbb{N}$ such that $r \geqslant 3$ and $r$ divides $n \geqslant 2 r$. Suppose that $D \in \mathbb{N}$ such that $n / r \leqslant D \leqslant n /(r-1)$. Then

$$
f(n, r, D)=\left(\begin{array}{c}
n / r+1 \\
2
\end{array}\right) .
$$


The following simple consequence of Turán's theorem will be used in the proof of Theorem 5 .

Fact 14. Let $n, r \in \mathbb{N}$ such that $r \leqslant n$. Then

$$
e(T(n, r)) \leqslant\left(1-\frac{1}{r}\right) \frac{n^{2}}{2} \text { and thus } e(\bar{T}(n, r)) \geqslant \frac{n^{2}}{2 r}-\frac{n}{2} .
$$

We will also require the following easy result.

Lemma 15. Let $n, r \in \mathbb{N}$ such that $r \geqslant 4$ and $r$ divides $n \geqslant 3 r$. Suppose that $D \in \mathbb{N}$ such that $n / r \leqslant D<(n+r) /(r-1)$. Then

$$
f(n, r, D)=\left(\begin{array}{c}
n / r+1 \\
2
\end{array}\right)
$$

\section{Proof of Theorem 5}

\subsection{Preliminaries}

Suppose for a contradiction that the result is false. Let $G$ be a counterexample with the fewest vertices. That is, $n=|V(G)|=r k$ for some $k \in \mathbb{N}, \Delta(G) \leqslant D$ for some $D \in \mathbb{N}$ such that $n / r \leqslant D \leqslant n-r, e(G)<f(n, r, D)$ and $G$ has no equitable $n / r$-colouring. By the Hajnal-Szemerédi theorem, $\Delta(G) \geqslant n / r$. Notice that given fixed $n$ and $r, f(n, r, D)$ is non-increasing with respect to $D$. Thus, we may assume that $\Delta(G)=D$.

We first show that $k \geqslant 4$. Indeed, if $n=2 r$ then $f(n, r, D) \leqslant\left(\begin{array}{l}3 \\ 2\end{array}\right)=3$. But it is easy to see that every graph $G_{1}$ on $2 r$ vertices and with $e\left(G_{1}\right) \leqslant 2$ has an equitable 2colouring. If $n=3 r$ then $f(n, r, D) \leqslant\left(\begin{array}{l}4 \\ 2\end{array}\right)=6$. Consider any graph $G_{1}$ on $3 r$ vertices with $e\left(G_{1}\right) \leqslant 5$ and $3 \leqslant \Delta\left(G_{1}\right) \leqslant 5$. Let $x$ denote the vertex in $G_{1}$ where $d_{G_{1}}(x)=\Delta\left(G_{1}\right)$. Since $3 \leqslant d_{G_{1}}(x) \leqslant 5, x$ lies in an independent set $I$ in $G_{1}$ of size $r$. But then $G_{1}-I$ contains $2 r$ vertices and at most 2 edges. So $G_{1}-I$ has an equitable 2-colouring and hence $G_{1}$ has an equitable 3-colouring.

Let $v \in V(G)$ such that $d_{G}(v)=D$. Set $G^{*}:=G-\left(N_{G}(v) \cup\{v\}\right)$. Since $f(n, r, D) \leqslant$ $D+e(\bar{T}(n-D-1, r-2))$ we have that $e\left(G^{*}\right)<e(\bar{T}(n-D-1, r-2))$. Thus, by Turán's theorem, $G^{*}$ contains an independent set of size $r-1$. Hence, $v$ lies in an independent set in $G$ of size $r$. Amongst all such independent sets of size $r$ that contain $v$, choose a set $I=\left\{v, x_{1}, \ldots, x_{r-1}\right\}$ such that $d_{G}\left(x_{1}\right)+\cdots+d_{G}\left(x_{r-1}\right)$ is maximised.

Set $G^{\prime}:=G-I, n^{\prime}:=\left|V\left(G^{\prime}\right)\right|=n-r$ and $D^{\prime}:=\Delta\left(G^{\prime}\right) \leqslant D$. Notice that $D^{\prime} \geqslant n^{\prime} / r$. (Indeed, if not, then by the Hajnal-Szemerédi theorem $G^{\prime}$ contains an equitable $n^{\prime} / r$ colouring. Thus, as $I$ is an independent set in $G$ this gives us an equitable $n / r$-colouring of $G$, a contradiction.) Furthermore, $D^{\prime} \leqslant n^{\prime}-r$. If not then

$$
e(G) \geqslant D+D^{\prime} \geqslant 2 D^{\prime} \geqslant 2\left(n^{\prime}-r+1\right)=2 n-4 r+2
$$


and further,

$$
\begin{aligned}
e(G) & <f(n, r, D) \leqslant f(n, r, n-2 r+1) \leqslant(n-2 r+1)+e(\bar{T}(2 r-2, r-2)) \\
& \leqslant(n-2 r+1)+(r+3)=n-r+4 .
\end{aligned}
$$

Therefore, $2 n-4 r+2<n-r+4$ and so $n<3 r+2$ a contradiction since $n=k r \geqslant 4 r$.

Since $n^{\prime} / r \leqslant D^{\prime} \leqslant n^{\prime}-r$, if $e\left(G^{\prime}\right)<f\left(n^{\prime}, r, D^{\prime}\right)$ then the minimality of $G$ implies that $G^{\prime}$ has an equitable $n^{\prime} / r$-colouring. This then implies that $G$ has an equitable $n / r$ colouring, a contradiction. Thus,

$$
e\left(G^{\prime}\right) \geqslant f\left(n^{\prime}, r, D^{\prime}\right)
$$

We now split our argument into three cases.

3.2 Case 1: $\mathbf{f}\left(\mathbf{n}^{\prime}, \mathbf{r}, \mathbf{D}^{\prime}\right)=\left(\begin{array}{c}\mathbf{n}^{\prime} / \mathbf{r}+1 \\ 2\end{array}\right)$.

By $(1), e\left(G^{\prime}\right) \geqslant\left(\begin{array}{c}n^{\prime} / r+1 \\ 2\end{array}\right)=\left(\begin{array}{c}n / r \\ 2\end{array}\right)$. Since $d_{G}(v)=D \geqslant n / r$,

$$
e(G) \geqslant \frac{n}{r}+\left(\begin{array}{c}
n / r \\
2
\end{array}\right)=\left(\begin{array}{c}
n / r+1 \\
2
\end{array}\right) \geqslant f(n, r, D)
$$

a contradiction, as desired.

\subsection{Case 2: $\mathbf{D}^{\prime} \leqslant \mathrm{D}-1$ and $\mathbf{f}\left(\mathbf{n}^{\prime}, \mathbf{r}, \mathbf{D}^{\prime}\right)=\mathbf{D}^{\prime}+\mathbf{e}\left(\overline{\mathbf{T}}\left(\mathbf{n}^{\prime}-\mathbf{D}^{\prime}-1, \mathbf{r}-2\right)\right)$.}

The following claim will be useful.

Claim 16. $D^{\prime}<\frac{r-1}{2 r-3} n-\frac{\left(r^{2}-r+1\right)}{2 r-3}$.

Proof. Note that

$$
D+D^{\prime}+e\left(\bar{T}\left(n^{\prime}-D^{\prime}-1, r-2\right)\right) \stackrel{(1)}{\leqslant} e(G)<f(n, r, D) \leqslant D+e(\bar{T}(n-D-1, r-2)) .
$$

Since $D^{\prime} \leqslant D-1$, clearly $e\left(\bar{T}\left(n^{\prime}-D, r-2\right)\right) \leqslant e\left(\bar{T}\left(n^{\prime}-D^{\prime}-1, r-2\right)\right)$. Thus, (2) implies that

$$
D^{\prime}+e\left(\bar{T}\left(n^{\prime}-D, r-2\right)\right)<e(\bar{T}(n-D-1, r-2)) .
$$

One can obtain $\bar{T}(n-D-1, r-2)$ from $\bar{T}\left(n^{\prime}-D, r-2\right)$ by adding $r-1$ vertices and at most

$$
\left(n^{\prime}-D\right)+\frac{n-D-2}{r-2} \text { edges. }
$$


Hence (3) and (4) give

$$
D^{\prime}<n^{\prime}-D+\frac{n-D-2}{r-2} .
$$

Rearranging, and using that $D^{\prime} \leqslant D-1$ and $n^{\prime}=n-r$ we get that

$$
\left(2+\frac{1}{r-2}\right) D^{\prime}<\left(1+\frac{1}{r-2}\right) n-\frac{\left(r^{2}-r+1\right)}{r-2} .
$$

Thus,

$$
D^{\prime}<\frac{r-1}{2 r-3} n-\frac{\left(r^{2}-r+1\right)}{2 r-3}
$$

as desired.

Since we are in Case 2 we have that

$$
D^{\prime}+e\left(\bar{T}\left(n-r-D^{\prime}-1, r-2\right)\right) \leqslant\left(\begin{array}{c}
n^{\prime} / r+1 \\
2
\end{array}\right)=\left(\begin{array}{c}
n / r \\
2
\end{array}\right) .
$$

Notice that for fixed $n$ and $r, D^{\prime}+e\left(\bar{T}\left(n-r-D^{\prime}-1, r-2\right)\right)$ is non-increasing as $D^{\prime}$ increases. Hence, (5) and Claim 16 imply that

$$
D^{\prime \prime}+e\left(\bar{T}\left(n-r-D^{\prime \prime}-1, r-2\right)\right) \leqslant \frac{n^{2}}{2 r^{2}}-\frac{n}{2 r}
$$

where $D^{\prime \prime}:=\left\lfloor(r-1) n /(2 r-3)-\left(r^{2}-r+1\right) /(2 r-3)\right\rfloor$. Note that

$$
n-r-\frac{r-1}{2 r-3} n+\frac{\left(r^{2}-r+1\right)}{2 r-3}-1=\frac{r-2}{2 r-3} n+\frac{4-r^{2}}{2 r-3} .
$$

So Fact 14 and (6) imply that

$$
\begin{aligned}
& \left(\frac{r-1}{2 r-3} n-\frac{\left(r^{2}-r+1\right)}{2 r-3}-\frac{(2 r-4)}{2 r-3}\right)+\frac{1}{2(r-2)}\left(\frac{r-2}{2 r-3} n+\frac{4-r^{2}}{2 r-3}\right)^{2} \\
& -\frac{1}{2}\left(\frac{r-2}{2 r-3} n+\frac{4-r^{2}}{2 r-3}\right) \leqslant \frac{n^{2}}{2 r^{2}}-\frac{n}{2 r} .
\end{aligned}
$$

Next we will move all terms from the previous equation to the left hand side and simplify. The coefficient of $n^{2}$ is

$$
\frac{r-2}{2(2 r-3)^{2}}-\frac{1}{2 r^{2}}=\frac{r^{3}-6 r^{2}+12 r-9}{2 r^{2}(2 r-3)^{2}}
$$

The coefficient of $n$ is

$$
\frac{r-1}{2 r-3}-\frac{(r-2)}{2(2 r-3)}+\frac{1}{2 r}+\frac{\left(4-r^{2}\right)}{(2 r-3)^{2}}=\frac{r^{2}-4 r+9}{2 r(2 r-3)^{2}} .
$$


The constant term is

$$
-\frac{\left(r^{2}+r-3\right)}{2 r-3}+\frac{\left(r^{2}-4\right)^{2}}{2(r-2)(2 r-3)^{2}}+\frac{\left(r^{2}-4\right)}{2(2 r-3)}=\frac{-r^{4}+3 r^{3}+4 r^{2}-26 r+28}{2(r-2)(2 r-3)^{2}} .
$$

Since $n \geqslant 4 r,(7)-(9)$ imply that

$$
\frac{8\left(r^{3}-6 r^{2}+12 r-9\right)}{(2 r-3)^{2}}+\frac{2\left(r^{2}-4 r+9\right)}{(2 r-3)^{2}}+\frac{-r^{4}+3 r^{3}+4 r^{2}-26 r+28}{2(r-2)(2 r-3)^{2}} \leqslant 0 .
$$

Multiplying (10) by $2(r-2)(2 r-3)^{2}$ we get

$$
15 r^{4}-121 r^{3}+364 r^{2}-486 r+244 \leqslant 0
$$

This yields a contradiction, since it is easy to check that

$$
15 r^{4}-121 r^{3}+364 r^{2}-486 r+244>0
$$

for all $r \in \mathbb{N}$ such that $r \geqslant 3$.

\subsection{Case 3: $\mathbf{D}^{\prime}=\mathbf{D}$ and $\mathbf{f}\left(\mathbf{n}^{\prime}, \mathbf{r}, \mathbf{D}^{\prime}\right)=\mathbf{D}^{\prime}+\mathbf{e}\left(\overline{\mathbf{T}}\left(\mathbf{n}^{\prime}-\mathbf{D}^{\prime}-1, \mathbf{r}-2\right)\right)$.}

By (1) we have that

$$
e\left(G^{\prime}\right) \geqslant f\left(n^{\prime}, r, D^{\prime}\right)=D^{\prime}+e\left(\bar{T}\left(n^{\prime}-D^{\prime}-1, r-2\right)\right) .
$$

Consider any vertex $x \in V\left(G^{\prime}\right)$ such that $d_{G^{\prime}}(x)=D^{\prime}=D$. Since $\Delta(G)=D, x$ is not adjacent to any vertex in $I=\left\{v, x_{1}, \ldots, x_{r-1}\right\}$. Further, $I$ was chosen such that $d_{G}\left(x_{1}\right)+\cdots+d_{G}\left(x_{r-1}\right)$ is maximised. Thus, $d_{G}\left(x_{1}\right)=\cdots=d_{G}\left(x_{r-1}\right)=D$. Together with (11) this implies that

$$
e(G) \geqslant(r+1) D+e\left(\bar{T}\left(n^{\prime}-D-1, r-2\right)\right) .
$$

Since $e(G)<f(n, r, D) \leqslant D+e(\bar{T}(n-D-1, r-2))$, (12) implies that

$$
r D+e\left(\bar{T}\left(n^{\prime}-D-1, r-2\right)\right)<e(\bar{T}(n-D-1, r-2)) .
$$

One can obtain $\bar{T}(n-D-1, r-2)$ from $\bar{T}\left(n^{\prime}-D-1, r-2\right)$ by adding $r$ vertices and at most

$$
\left(n^{\prime}-D-1\right)+\frac{2(n-D-3)}{r-2}+1 \text { edges. }
$$

Thus, (13) and (14) imply that

$$
r D<n-r-D+\frac{2(n-D-3)}{r-2}
$$


and so

$$
\left(r+1+\frac{2}{r-2}\right) D<\left(1+\frac{2}{r-2}\right) n+\frac{\left(-r^{2}+2 r-6\right)}{r-2}<\left(1+\frac{2}{r-2}\right) n .
$$

If $r=3$ then (15) implies that

$$
D<\frac{n}{2}
$$

Since $f\left(n^{\prime}, 3, D\right)=\min \left\{\left(\begin{array}{c}n^{\prime} / 3+1 \\ 2\end{array}\right), D+\left(\begin{array}{c}n^{\prime}-D-1 \\ 2\end{array}\right)\right\}$ it is easy to see that if $f\left(n^{\prime}, 3, D\right)=$ $D+\left(\begin{array}{c}n^{\prime}-D-1 \\ 2\end{array}\right)$ then $D \geqslant 2 n^{\prime} / 3+1=2 n / 3-1$. Thus, $2 n / 3-1 \leqslant D<n / 2$, a contradiction since $n \geqslant 4 r=12$.

If $r \geqslant 4$ then (15) implies that

$$
D<\frac{n}{r-1}=\frac{n^{\prime}}{r-1}+\frac{r}{r-1}
$$

Since $n^{\prime} \geqslant 3 r$, Lemma 15 implies that $f\left(n^{\prime}, r, D^{\prime}\right)=\left(\begin{array}{c}n^{\prime} / r+1 \\ 2\end{array}\right)$ and so we are in Case 1 , which we have already dealt with.

\section{The extremal examples for Conjecture 7}

Proposition 17. Suppose that $n, r, k \in \mathbb{N}$ such that $r \geqslant 2$ divides $n$ and $1 \leqslant k<n / r$. Then there exists a graph $G$ on $n$ vertices whose degree sequence $d_{1} \leqslant \ldots \leqslant d_{n}$ satisfies

- $d_{i}=(r-2) n / r+k-1$ for all $1 \leqslant i \leqslant k$;

- $d_{i}=(r-1) n / r$ for all $k+1 \leqslant i \leqslant(r-2) n / r+k$;

- $d_{i}=n-k-1$ for all $(r-2) n / r+k+1 \leqslant i \leqslant n-k+1$;

- $d_{i}=n-1$ for all $n-k+2 \leqslant i \leqslant n$,

but such that $G$ does not contain a perfect $K_{r}$-packing.

Proof. Let $G^{\prime}$ denote the complete $(r-2)$-partite graph whose vertex classes $V_{1}, \ldots, V_{r-2}$ each have size $n / r$. Obtain $G$ from $G^{\prime}$ by adding the following vertices and edges: Add a set $V_{r-1}$ of $2 n / r-2 k+1$ vertices to $G^{\prime}$, a set $V_{r}$ of $k-1$ vertices and a set $V_{0}$ of $k$ vertices. Add all edges from $V_{0} \cup V_{r-1} \cup V_{r}$ to $V_{1} \cup \cdots \cup V_{r-2}$. Further, add all edges with both endpoints in $V_{r-1} \cup V_{r}$. Add all possible edges between $V_{0}$ and $V_{r}$.

So $V_{0}$ is an independent set, and there are no edges between $V_{0}$ and $V_{r-1}$. This implies that any copy of $K_{r}$ in $G$ containing a vertex from $V_{0}$ must also contain at least one vertex from $V_{r}$. But since $\left|V_{0}\right|>\left|V_{r}\right|$ this implies that $G$ does not contain a perfect $K_{r}$-packing. Furthermore, $G$ has our desired degree sequence. 
Notice that the graphs $G$ considered in Proposition 17 satisfy $(\beta)$ from Conjecture 7 and only fail to satisfy $(\alpha)$ in the case when $i=k$ (and in this case $d_{k}=(r-2) n / r+k-1$ ).

Let $n, r \in \mathbb{N}$ such that $r$ divides $n$. Denote by $T^{*}(n, r)$ the complete $r$-partite graph on $n$ vertices with $r-2$ vertex classes of size $n / r$, one vertex class of size $n / r-1$ and one vertex class of size $n / r+1$. Then $T^{*}(n, r)$ does not contain a perfect $K_{r}$-packing. Furthermore, $T^{*}(n, r)$ satisfies $(\alpha)$ but condition $(\beta)$ fails; we have that $d_{n / r+1}=(r-1) n / r-1$ here. Thus, together $T^{*}(n, r)$ and Proposition 17 show that, if true, Conjecture 7 is essentially best possible.

\section{$5 \quad$ A special case of Conjecture 7}

We now give a simple proof of Conjecture 7 in the case when $G$ is $K_{r+1}$-free.

Theorem 18. Let $n, r \in \mathbb{N}$ such that $r \geqslant 2$ divides $n$. Suppose that $G$ is a graph on $n$ vertices with degree sequence $d_{1} \leqslant \ldots \leqslant d_{n}$ such that:

- $d_{i} \geqslant(r-2) n / r+i$ for all $i<n / r$;

- $d_{n / r+1} \geqslant(r-1) n / r$.

Further suppose that no vertex $x \in V(G)$ of degree less than $(r-1) n / r$ lies in a copy of $K_{r+1}$. Then $G$ contains a perfect $K_{r}$-packing.

Proof. We prove the theorem by induction on $n$. In the case when $n=r$ then $d_{n / r+1}=$ $d_{2} \geqslant(r-1) r / r=r-1$. This implies that every vertex in $G$ has degree $r-1$. Hence $G=K_{r}$ as desired. So suppose that $n>r$ and the result holds for smaller values of $n$. Let $x_{1} \in V(G)$ such that $d_{G}\left(x_{1}\right)=d_{1} \geqslant(r-2) n / r+1$. If $d_{G}\left(x_{1}\right) \geqslant(r-1) n / r$ then $\delta(G) \geqslant(r-1) n / r$. Thus $G$ contains a perfect $K_{r}$-packing by the Hajnal-Szemerédi theorem. So we may assume that $(r-2) n / r+1 \leqslant d_{G}\left(x_{1}\right)<(r-1) n / r$. In particular, $x_{1}$ does not lie in a copy of $K_{r+1}$. We first find a copy of $K_{r}$ containing $x_{1}$. If $r=2, x_{1}$ has a neighbour and so we have our desired copy of $K_{2}$. So assume that $r \geqslant 3$. Certainly $N_{G}\left(x_{1}\right)$ contains a vertex $x_{2}$ such that $d_{G}\left(x_{2}\right) \geqslant(r-1) n / r$. Thus, $\left|N_{G}\left(x_{1}\right) \cap N_{G}\left(x_{2}\right)\right| \geqslant$ $(r-3) n / r+1>0$. So if $r=3$ we obtain our desired copy of $K_{r}$. Otherwise, we can find a vertex $x_{3} \in N_{G}\left(x_{1}\right) \cap N_{G}\left(x_{2}\right)$ such that $d_{G}\left(x_{3}\right) \geqslant(r-1) n / r$. We can repeat this argument until we have obtained vertices $x_{1}, \ldots, x_{r}$ that together form a copy $K_{r}^{\prime}$ of $K_{r}$.

Let $G^{\prime}:=G-V\left(K_{r}^{\prime}\right)$ and set $n^{\prime}:=n-r=\left|V\left(G^{\prime}\right)\right|$. Since $G$ does not contain a copy of $K_{r+1}$ containing $x_{1}$, every vertex $x \in V(G) \backslash V\left(K_{r}^{\prime}\right)$ sends at most $r-1$ edges to $K_{r}^{\prime}$ in $G$. Thus, $d_{G^{\prime}}(x) \geqslant d_{G}(x)-(r-1)$ for all $x \in V\left(G^{\prime}\right)$. So if $d_{G}(x) \geqslant(r-1) n / r$ then $d_{G^{\prime}}(x) \geqslant(r-1) n / r-(r-1)=(r-1) n^{\prime} / r$ for all $x \in V\left(G^{\prime}\right)$. If a vertex $y \in V\left(G^{\prime}\right)$ does not lie in a copy of $K_{r+1}$ in $G$ then clearly $y$ does not lie in a copy of $K_{r+1}$ in $G^{\prime}$. This means that no vertex $y \in V\left(G^{\prime}\right)$ of degree less than $(r-1) n^{\prime} / r$ lies in a copy of $K_{r+1}$.

Let $d_{1}^{\prime} \leqslant \ldots \leqslant d_{n^{\prime}}^{\prime}$ denote the degree sequence of $G^{\prime}$. It is easy to check that $d_{i}^{\prime} \geqslant$ $(r-2) n^{\prime} / r+i$ for all $i<n^{\prime} / r$ and that $d_{n^{\prime} / r+1}^{\prime} \geqslant(r-1) n^{\prime} / r$. Indeed, since $x_{1} \in V\left(K_{r}^{\prime}\right)$ 
where $d_{G}\left(x_{1}\right)=d_{1}$, we have that $d_{i}^{\prime} \geqslant d_{i+1}-(r-1)$ for all $1 \leqslant i \leqslant n^{\prime}$. Thus, for all $1 \leqslant i<n^{\prime} / r=n / r-1, d_{i}^{\prime} \geqslant d_{i+1}-(r-1) \geqslant(r-2) n / r+(i+1)-(r-1)=(r-2) n^{\prime} / r+i$. Similarly, $d_{n^{\prime} / r+1}^{\prime}=d_{n / r}^{\prime} \geqslant d_{n / r+1}-(r-1) \geqslant(r-1) n / r-(r-1)=(r-1) n^{\prime} / r$. Hence, by induction $G^{\prime}$ contains a perfect $K_{r}$-packing. Together with $K_{r}^{\prime}$ this gives us our desired perfect $K_{r}$-packing in $G$.

\section{Proof of Theorem 10}

Consider any $x_{1} \in V(G)$ such that $d_{G}\left(x_{1}\right) \geqslant(r-1) n / r$. Since $d_{n / r} \geqslant(r-1) n / r$ we can greedily select vertices $x_{2}, \ldots, x_{r-1}$ such that

- $x_{1}, \ldots, x_{r-1}$ induce a copy of $K_{r-1}$ in $G$;

- $d_{G}\left(x_{i}\right) \geqslant(r-1) n / r$ for all $1 \leqslant i \leqslant r-1$.

Note that since $G$ is $K_{r+1}$-free, $\cap_{i=1}^{r-1} N_{G}\left(x_{i}\right)$ is an independent set. The choice of $x_{1}, \ldots$, $x_{r-1}$ implies that $\left|\cap_{i=1}^{r-1} N_{G}\left(x_{i}\right)\right| \geqslant n / r$. Let $V_{1}$ denote a subset of $\cap_{i=1}^{r-1} N_{G}\left(x_{i}\right)$ of size $n / r$. Thus $V_{1}$ contains a vertex $x_{1}^{1}$ of degree at least $(r-1) n / r$.

As before we can find vertices $x_{2}^{1}, \ldots, x_{r-1}^{1}$ such that

- $x_{1}^{1}, \ldots, x_{r-1}^{1}$ induce a copy of $K_{r-1}$ in $G$;

- $d_{G}\left(x_{i}^{1}\right) \geqslant(r-1) n / r$ for all $1 \leqslant i \leqslant r-1$.

So $\cap_{i=1}^{r-1} N_{G}\left(x_{i}^{1}\right)$ is an independent set of size at least $n / r$. Let $V_{2}$ denote a subset of $\cap_{i=1}^{r-1} N_{G}\left(x_{i}^{1}\right)$ of size $n / r$. Note that $N_{G}\left(x_{1}^{1}\right) \cap V_{1}=\emptyset$ since $x_{1}^{1} \in V_{1}$ and $V_{1}$ is an independent set. Thus as $V_{2} \subseteq N_{G}\left(x_{1}^{1}\right), V_{1} \cap V_{2}=\emptyset$.

Our aim is to find disjoint sets $V_{1}, \ldots, V_{r} \subseteq V(G)$ of size $n / r$ and vertices $x_{1}^{1}, \ldots, x_{r-1}^{1}$, $x_{1}^{2}, \ldots, x_{r-1}^{2}, \ldots, x_{1}^{r-1}, \ldots, x_{r-1}^{r-1}$ with the following properties:

- $G\left[V_{j}\right]$ is an independent set for all $1 \leqslant j \leqslant r$;

- Given any $1 \leqslant j \leqslant r-1, x_{k}^{j} \in V_{k}$ for each $1 \leqslant k \leqslant j$;

- $d_{G}\left(x_{k}^{j}\right) \geqslant(r-1) n / r$ for all $1 \leqslant j \leqslant r-1$ and $1 \leqslant k \leqslant r-1$;

- $x_{1}^{j}, \ldots, x_{r-1}^{j}$ induce a copy of $K_{r-1}$ in $G$ for all $1 \leqslant j \leqslant r-1$.

Clearly finding such a partition $V_{1}, \ldots, V_{r}$ of $V(G)$ implies that $G \subseteq T(n, r)$.

Suppose that for some $1<j<r$ we have defined sets $V_{1}, \ldots, V_{j}$ and vertices $x_{1}^{1}, \ldots, x_{r-1}^{1}, \ldots, x_{1}^{j-1}, \ldots, x_{r-1}^{j-1}$ with our desired properties. Since $d_{n / r} \geqslant(r-1) n / r$ and $V_{1}, \ldots, V_{j}$ are independent sets of size $n / r$ we can choose vertices $x_{1}^{j}, \ldots, x_{j}^{j}$ such that for all $1 \leqslant k \leqslant j$

- $x_{k}^{j} \in V_{k}$ and $d_{G}\left(x_{k}^{j}\right) \geqslant(r-1) n / r$. 
This degree condition, together with the fact that $x_{1}^{j}, \ldots, x_{j}^{j}$ lie in different vertex classes, implies that these vertices form a copy of $K_{j}$ in $G$. We now greedily select further vertices $x_{j+1}^{j}, \ldots, x_{r-1}^{j}$ such that

- $x_{1}^{j}, \ldots, x_{r-1}^{j}$ induce a copy of $K_{r-1}$ in $G$;

- $d_{G}\left(x_{k}^{j}\right) \geqslant(r-1) n / r$ for all $j+1 \leqslant k \leqslant r-1$.

So $\cap_{i=1}^{r-1} N_{G}\left(x_{i}^{j}\right)$ is an independent set of size at least $n / r$. Let $V_{j+1}$ denote a subset of $\cap_{i=1}^{r-1} N_{G}\left(x_{i}^{j}\right)$ of size $n / r$. Note that, for each $1 \leqslant k \leqslant j, N_{G}\left(x_{k}^{j}\right) \cap V_{k}=\emptyset$ since $x_{k}^{j} \in V_{k}$ and $V_{k}$ is an independent set. Thus as $V_{j+1} \subseteq N_{G}\left(x_{k}^{j}\right)$ for each $1 \leqslant k \leqslant j, V_{j+1}$ is disjoint from $V_{1} \cup \cdots \cup V_{j}$.

Repeating this argument we obtain our desired sets $V_{1}, \ldots, V_{r} \subseteq V(G)$ and vertices $x_{1}^{1}, \ldots, x_{r-1}^{1}, x_{1}^{2}, \ldots, x_{r-1}^{2}, \ldots, x_{1}^{r-1}, \ldots, x_{r-1}^{r-1}$.

\section{Acknowledgements}

We thank the referees for their comments. In particular, we thank one referee for pointing out the work in [1], [5] and [9].

This research was carried out whilst the third author was visiting the Department of Mathematics of the University of Illinois at Urbana-Champaign. This author would like to thank the department for the hospitality he received. We would also like to thank Hal Kierstead for helpful discussions.

\section{References}

[1] A. Akiyama and P. Frankl, On the Size of Graphs with Complete-Factors, J. Graph Theory 9 (1985), 197-201.

[2] P. Allen, J. Böttcher, J. Hladký and D. Piguet, A density Corrádi-Hajnal theorem, Electronic Notes in Discrete Mathematics 38 (2011), 31-36.

[3] V. Chvátal, On Hamilton's ideals, J. Combin. Theory B 12 (1972), 163-168.

[4] K. Corrádi and A. Hajnal, On the maximal number of independent circuits in a graph, Acta Math. Acad. Sci. Hungar. 14 (1964), 423-439.

[5] G.A. Dirac, Structural properties and circuits in graphs, in: C.St.-J.A. NashWilliams, J. Sheehan (Eds.), Proceedings of the 5th British Combinatorial Conference, Congressus Numerantium, No. XV, Utilitas Math., Winnipeg, Man., 1976, pp. $135-140$.

[6] P. Erdős, On the graph theorem of Turán, Mat. Lapok. 21 (1970), 249-251.

[7] A. Hajnal and E. Szemerédi, Proof of a conjecture of Erdös, Combinatorial Theory and its Applications vol. II 4 (1970), 601-623.

[8] P. Hell and D.G. Kirkpatrick, On the complexity of general graph factor problems, SIAM J. Computing 12 (1983), 601-609. 
[9] P. Justesen, On independent circuits in finite graphs and a conjecture of Erdős and Pósa, Ann. Discrete Math. 41 (1989), 299-306

[10] H.A. Kierstead and A.V. Kostochka, An Ore-type Theorem on Equitable Coloring, J. Combin. Theory B 98 (2008), 226-234.

[11] F. Knox and A. Treglown, Embedding spanning bipartite graphs of small bandwidth, Combin. Probab. Comput. 22 (2013), 71-96.

[12] D. Kühn and D. Osthus, Critical chromatic number and the complexity of perfect packings in graphs, 17th ACM-SIAM Symposium on Discrete Algorithms (SODA 2006), 851-859.

[13] D. Kühn and D. Osthus, The minimum degree threshold for perfect graph packings, Combinatorica 29 (2009), 65-107.

[14] D. Kühn, D. Osthus and A. Treglown, An Ore-type theorem for perfect packings in graphs, SIAM J. Disc. Math. 23 (2009), 1335-1355.

\section{Appendix}

Here we give proofs of Proposition 13 and Lemma 15. The following fact will be used in both of these proofs.

Fact 19. Fix $n, r \in \mathbb{N}$ such that $r \geqslant 3$ and $r$ divides $n \geqslant 2 r$. Define

$$
h(x):=x+\frac{(n-x-1)^{2}}{2(r-2)}-\frac{1}{2}(n-x-1) .
$$

Then $h(x)$ is a decreasing function for $x \in[0, n /(r-1)]$. Moreover, if $n \geqslant 3 r$ then $h(x)$ is a decreasing function for $x \in[0,(n+r) /(r-1)]$.

Proof. Notice that

$$
h^{\prime}(x)=\frac{3}{2}-\frac{(n-x-1)}{r-2}=\frac{x}{r-2}+\frac{1-n}{r-2}+\frac{3}{2} .
$$

So for $x \leqslant n /(r-1)$,

$$
h^{\prime}(x) \leqslant \frac{n}{(r-1)(r-2)}+\frac{1-n}{r-2}+\frac{3}{2}=-\frac{n}{r-1}+\frac{1}{r-2}+\frac{3}{2} .
$$

Note that $3(r-1) / 2+(r-1) /(r-2)<n$ since $n \geqslant 2 r$ and $r \geqslant 3$. Thus,

$$
h^{\prime}(x) \leqslant-\frac{n}{r-1}+\frac{1}{r-2}+\frac{3}{2}<0 .
$$

If $x \leqslant(n+r) /(r-1)$ then

$$
h^{\prime}(x) \leqslant \frac{n+r}{(r-1)(r-2)}+\frac{1-n}{r-2}+\frac{3}{2}=-\frac{n}{r-1}+\frac{1}{r-2}+\frac{r}{(r-1)(r-2)}+\frac{3}{2} .
$$


If $n \geqslant 3 r$ then $n>3 r / 2+4$. So $n>3(r-1) / 2+(2 r-1) /(r-2)$. Thus,

$$
h^{\prime}(x) \leqslant-\frac{n}{r-1}+\frac{1}{r-2}+\frac{r}{(r-1)(r-2)}+\frac{3}{2}<0,
$$

as desired.

Proof of Proposition 13. We need to show that, for all $D \in \mathbb{N}$ such that $n / r \leqslant D \leqslant$ $n /(r-1)$,

$$
\frac{n^{2}}{2 r^{2}}+\frac{n}{2 r}=\left(\begin{array}{c}
n / r+1 \\
2
\end{array}\right) \leqslant D+e(\bar{T}(n-D-1, r-2)) .
$$

Since $D \leqslant n /(r-1)$, Facts 14 and 19 imply that

$$
\begin{aligned}
D+e(\bar{T}(n-D-1, r-2)) & \geqslant D+\frac{(n-D-1)^{2}}{2(r-2)}-\frac{(n-D-1)}{2} \\
& \geqslant \frac{n}{r-1}+\frac{1}{2(r-2)}\left[\frac{(r-2)}{r-1} n-1\right]^{2}-\frac{1}{2}\left[\frac{(r-2)}{r-1} n-1\right] \\
& \geqslant \frac{(r-2)}{2(r-1)^{2}} n^{2}-\frac{(r-2)}{2(r-1)} n .
\end{aligned}
$$

Thus, it suffices to show that

$$
\frac{(r-2)}{2(r-1)^{2}} n-\frac{r-2}{2(r-1)} \geqslant \frac{n}{2 r^{2}}+\frac{1}{2 r}
$$

Notice that

$$
\frac{r-2}{2(r-1)^{2}}-\frac{1}{2 r^{2}}=\frac{(r-2) r^{2}-(r-1)^{2}}{2 r^{2}(r-1)^{2}}=\frac{r^{3}-3 r^{2}+2 r-1}{2 r^{2}(r-1)^{2}}
$$

and

$$
\frac{r-2}{2(r-1)}+\frac{1}{2 r}=\frac{r^{2}-r-1}{2 r(r-1)} .
$$

Since $n \geqslant 2 r,(16)$ implies that it suffices to show that

$$
\frac{r^{3}-3 r^{2}+2 r-1}{r(r-1)^{2}}-\frac{r^{2}-r-1}{2 r(r-1)} \geqslant 0 .
$$

Note that $r^{3} \geqslant 4 r^{2}-4 r+3$ as $r \geqslant 3$. Thus, $2\left(r^{3}-3 r^{2}+2 r-1\right) \geqslant\left(r^{2}-r-1\right)(r-1)$. So indeed (18) is satisfied, as desired.

Proof of Lemma 15. We need to show that, for all $D \in \mathbb{N}$ such that $n / r \leqslant D<$ $(n+r) /(r-1)$,

$$
\frac{n^{2}}{2 r^{2}}+\frac{n}{2 r}=\left(\begin{array}{c}
n / r+1 \\
2
\end{array}\right) \leqslant D+e(\bar{T}(n-D-1, r-2))
$$


Since $D<(n+r) /(r-1)$ we have that $D \leqslant n /(r-1)+1$. So Facts 14 and 19 imply that

$$
\begin{aligned}
D+e(\bar{T}(n-D-1, r-2)) & \geqslant D+\frac{(n-D-1)^{2}}{2(r-2)}-\frac{(n-D-1)}{2} \\
& \geqslant \frac{n}{r-1}+1+\frac{1}{2(r-2)}\left[\frac{(r-2)}{r-1} n-2\right]^{2}-\frac{1}{2}\left[\frac{(r-2)}{r-1} n-2\right] \\
& \geqslant \frac{(r-2)}{2(r-1)^{2}} n^{2}-\frac{(r-2)}{2(r-1)} n-\frac{n}{r-1} .
\end{aligned}
$$

Thus, it suffices to show that

$$
\frac{(r-2)}{2(r-1)^{2}} n-\frac{(r-2)}{2(r-1)}-\frac{1}{r-1} \geqslant \frac{n}{2 r^{2}}+\frac{1}{2 r} .
$$

Notice that

$$
\frac{r-2}{2(r-1)}+\frac{1}{r-1}+\frac{1}{2 r}=\frac{r^{2}+r-1}{2 r(r-1)} .
$$

Since $n \geqslant 3 r,(17)$ and (19) imply that it suffices to show that

$$
\frac{3\left(r^{3}-3 r^{2}+2 r-1\right)}{2 r(r-1)^{2}}-\frac{r^{2}+r-1}{2 r(r-1)} \geqslant 0
$$

Note that $2 r^{3}-9 r^{2}+8 r-4 \geqslant 0$ as $r \geqslant 4$. Thus, $3\left(r^{3}-3 r^{2}+2 r-1\right) \geqslant\left(r^{2}+r-1\right)(r-1)$. So indeed (20) is satisfied, as desired. 